\title{
Simulating and Evaluating Different Boarding Strategies on the Example of the Airbus A320
}

\author{
Jürgen Wunderlich*
}

Faculty of Computer Science, Landshut University of Applied Sciences, Am Lurzenhof 1, 84036 Landshut, Germany; *juergen.wunderlich@haw-landshut.de

SNE 31(4), 2021, 217-222, DOI: 10.11128/sne.31.tn.10585 Received: 2021-03-15 (Selected ASIM SST 2020 Postconf. Pub., English version); Revised: 2021-10-30; Accepted: 2021-11-15 SNE - Simulation Notes Europe, ARGESIM Publisher Vienna ISSN Print 2305-9974, Online 2306-0271, www.sne-journal.org

Abstract. Based on an Airbus A320 simulation model, this paper compares the boarding times of random boarding with the most frequently applied boarding strategy backto-front boarding as well as the alternatives outside-in boarding and back-to-front combined with outside-in boarding. The study shows that, on the one hand, outsidein boarding can reduce boarding times by more than $12 \%$, but on the other hand, this also requires a high degree of discipline. As boarding is part of the turnaround process, shorter boarding times mean faster take offs and allow an airport to handle a higher number of planes.

\section{Introduction}

Boarding strategies are the subject of ongoing discussions. As a result, travellers, airlines, and airports regularly develop ideas for improvement.

These ideas were rarely implemented pre-pandemic. However, current challenges posed by infection control and the results of the following simulation study may change this attitude.

\section{Motivation}

In times of COVID-19, many airlines have adjusted the boarding process to ensure that the minimum distance can be maintained [3]. This is an excellent opportunity to think about boarding strategies different from the most common one [2], which is to let passengers board the aircraft in groups, starting with the back rows after firstclass and other priority passengers have boarded.

Additionally, boarding is part of the turnaround process. This term denotes the handling of an aircraft between landing and takeoff and should be as efficient as possible, which becomes all the more important as air traffic increases again. The reason is that certain activities, such as safety instructions by flight attendants, cannot begin until boarding is completed. In this respect, several criteria and their interactions must be taken into account, for which simulation is a suitable approach.

\section{Objective}

The final goal of this study is to improve the flow and efficiency of the boarding process by selecting the most appropriate boarding method. The basic idea here is to avoid congestions in the aisle as much as possible by varying the sequence of boarding passengers, which should lead to an acceleration of boarding, hence to a reduction of the boarding time required and, consequently, to a shorter turnaround process time.

Since the turnaround process is similar for most aircraft types and only the sequence and duration of individual sub-processes differ, the present study will continue to be structured in such a way that the core concepts can easily be transferred to other aircraft types. For this purpose, a realistic reference system is first defined on the basis of which the simulation model is finally created and the advantages and disadvantages of each boarding strategy investigated are evaluated.

\section{Reference System}

With more than 15,000 aircraft sold, the A320 family is Airbus' greatest success [5]. Therefore, this aircraft type is chosen for this simulation. By default, its seats are divided into business and economy class and arranged in a configuration of 154 seats with 30 rows of seats, 2 of which are not intended for passengers. Of these, 28 seats in the first 7 rows are in Business Class and 126 seats in rows 8-30 are in Economy Class [4]. For passengers, a distinction is made between business travelers and tourists, or whether they carry hand luggage to be stowed in the overhead lockers. 
Furthermore, the reference system and subsequently the simulation also are based on the following eight assumptions:

- boarding begins when the passengers are called, at which time both the checked-in passengers and the aircraft are already at the gate

- each passenger already has a fixed seat, i.e. there is no free choice of seats

- the boarding pass control is carried out by the airport staff and ensures compliance with the order of boarding

- passengers enter the aircraft in the order of boarding through the front aircraft door via a passenger boarding bridge

- a single aisle is defined by the A320 aircraft type, which means that there is only one aisle available for moving through the aircraft

- on the plane passengers behave well and do not pass each other

- passengers have only one or no pieces of hand luggage, which already have the prescribed dimensions

- there is sufficient storage space for the hand luggage of each passenger, so that entering passengers can stow it without capacity-related time delays

The simplest boarding method used is random boarding, which is practiced, for example, by the airlines Lufthansa and Eurowings at Munich Airport. With the random boarding method, all passengers have a reserved seat, but can board the aircraft in random order without any further instructions. Only the individual booking classes (Zone 1 for Business and Zone 2 for Economy Class) are boarded one after the other.

This method is strikingly simple. An additional advantage is the distributed utilization of the aircraft aisle. Passengers do not crowd the same rows or the same overhead lockers all at once. With this boarding method traffic jams also occur - for example, because people have to rise from their seats repeatedly to let others pass - but these are at least spread over the entire aisle of the aircraft. A disadvantage of this method is that the order of boarding cannot be influenced.

The boarding process itself begins with boarding pass control. Passengers then pass through the buffer sleeve before reaching the first row of aisles. There they check to see if their seat is in it. If this is the case, they first stow their carry-on luggage - if any. This is followed by taking their seats.
The time required for this depends on the exact location of the seat and on how many seats in front of the assigned seat are already occupied. As long as a passenger has not reached his or her destination row, he or she continues to walk row by row until his or her seat is found. The exact process is shown in Figure 1. In the case of a business class passenger, only the time taken to occupy a seat changes or is shorter compared to an economy class passenger, as there are only two seats on each side in business class.

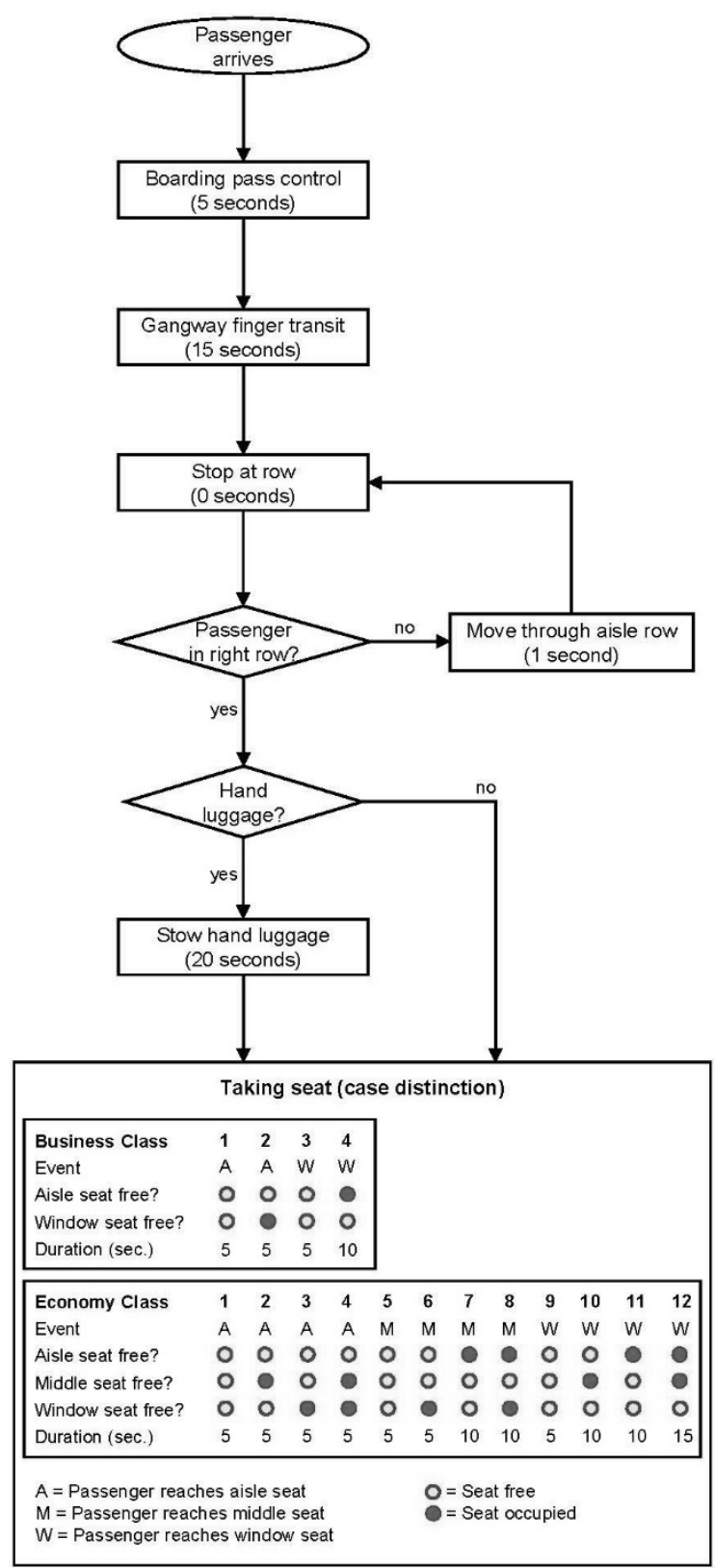

Figure 1: Flowchart of the boarding process from the perspective of a passenger. 
The times for the individual process steps were determined in cooperation with Martin Bertling, a process planner at Munich Airport. They are based on the official documentation for airport planners "AIRBUS A320 Aircraft Characteristics Airport And Maintenance Planning, Chapter 5-2-0" (Feb. 2018) [1]. To this, still missing data was added based on the doctoral dissertation "Analyse der Verzögerungen beim Boarding von Flugzeugen und Untersuchung möglicher Optimierungsansätze" by Holger Stefan Appel (2014) [2].

\begin{tabular}{|c|c|c|}
\hline Key figure & Time data / share & Source \\
\hline $\begin{array}{l}\text { average } \\
\text { boarding } \\
\text { time }\end{array}$ & 18 minutes & $\begin{array}{l}\text { Process planner Mu- } \\
\text { nich Airport, official } \\
\text { document for airport } \\
\text { planners }\end{array}$ \\
\hline $\begin{array}{l}\text { Boarding } \\
\text { pass control }\end{array}$ & 5 seconds & $\begin{array}{l}\text { Process planner Mu- } \\
\text { nich Airport }\end{array}$ \\
\hline $\begin{array}{l}\text { Entry time } \\
\text { into the air- } \\
\text { craft without } \\
\text { queueing }\end{array}$ & 15 seconds & $\begin{array}{l}\text { Process planner Mu- } \\
\text { nich Airport }\end{array}$ \\
\hline $\begin{array}{l}\text { Taking seat } \\
\text { (see fig. 1) }\end{array}$ & $\begin{array}{l}\text { no person: } 5 \mathrm{sec} \\
\text { one person: } 10 \mathrm{sec} \\
\text { two persons: } 15 \mathrm{sec} \text {. }\end{array}$ & $\begin{array}{l}\text { Process planner Mu- } \\
\text { nich Airport }\end{array}$ \\
\hline $\begin{array}{l}\text { Hand luggage } \\
\text { share }\end{array}$ & $\begin{array}{l}\text { Business travelers: } \\
95 \% \\
\text { Tourists: } 90 \%\end{array}$ & $\begin{array}{l}\text { Process planner Mu- } \\
\text { nich Airport, PhD } \\
\text { thesis Holger Appel }\end{array}$ \\
\hline $\begin{array}{l}\text { Hand luggage } \\
\text { time }\end{array}$ & 20 seconds & $\begin{array}{l}\text { Process planner Mu- } \\
\text { nich Airport, PhD } \\
\text { thesis Holger Appel }\end{array}$ \\
\hline
\end{tabular}

\section{Simulation Model}

The starting point for the simulation in Plant Simulation is the passenger list. This list determines the order of the passengers arriving at the boarding pass control and contains the time of entry, the assigned seat as well as the number of hand luggage items for each passenger, whereas the order of the passengers and the number or presence of hand luggage items are based on random numbers.

The aircraft type A320 is divided into 28 rows of seats that can be occupied, which are represented in the model as separate application modules. Each application module comprises either four (Business Class) or six (Economy Class) seats, which are replicated in the form of single stations. Both the occupancy of the seats and the passengers in the aisle or in a row of seats are illustrated with the aid of animations. Each passenger is inserted into the simulation model as a moveable unit. The arrival of the first passenger at boarding pass control marks the starting point of the simulation. The simulation is finished as soon as the last passenger has taken his or her seat.

The simulation model was validated against the total lead time for a boarding process. The average lead time for random boarding resulting from multiple simulation runs was 17 minutes and 43 seconds. This means that the deviation from the 18 minutes given by the process planner at Munich Airport as the average boarding time is only $1.57 \%$, which means that the model can be considered valid and thus forms a solid basis for the experiments.

Table 1: Data source for the boarding process.
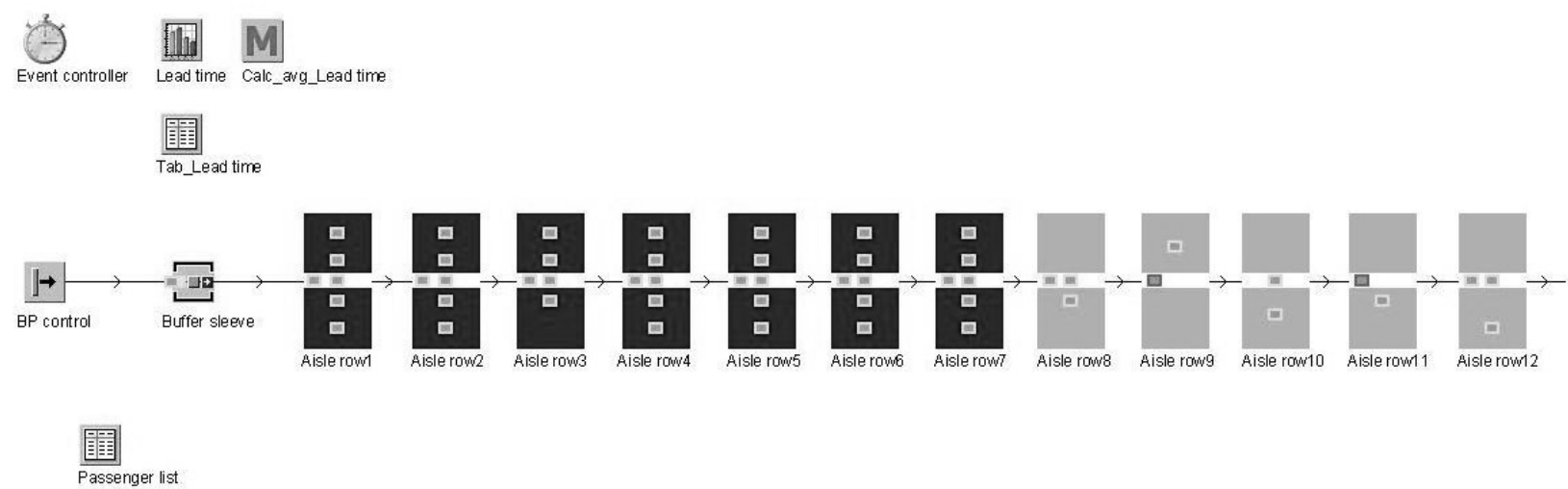

Figure 2: Simulating random boarding (excerpt). 


\section{Experiments}

As alternatives to random boarding, the boarding strategies back-to-front boarding, outside-in boarding, and the combination of back-to-front and outside-in boarding are mentioned in the literature. Therefore, after a brief explanation, a simulation-based investigation of these three methods is conducted.

The basic idea of back-to-front boarding is to let passengers board from the back to the front [2]. This is to prevent the rear section of the aisle from remaining temporarily unused at the start of boarding because of the first passengers blocking the aisle in the front section in order to stow their hand luggage. This means that the passengers in the back enter the aircraft first (with the exception of Business Class), so that each passenger can reach his or her seat with as few interruptions as possible. The simulation resulted in an average boarding time of 17 minutes and 55 seconds for back-to-front boarding, which means it is twelve seconds slower compared to random boarding.
With the outside-in boarding method, the plane is boarded from the outside to the inside, i.e. first the window seats, then the middle seats and finally the aisle seats [2]. It does not matter in which row the passengers are seated. However, business class passengers also enjoy higher priority and thus are boarded first. In the simulation, an average lead time of 15 minutes and 34 seconds was achieved for this boarding method. Compared to the initial value, this result yields an improvement of two minutes and nine seconds, or 12.1 percent.

Just as with the back-to-front boarding method, with the combination of back-to-front and outside-in boarding, the passengers enter the aircraft from the back to the front. At the same time, according to the principle of outside-in boarding, first the window seats, then the middle seats and finally the aisle seats are occupied [2]. Here, too, business class passengers are the first to board and economy class passengers follow. In the simulation runs, the average lead time for this boarding method was exactly 16 minutes.

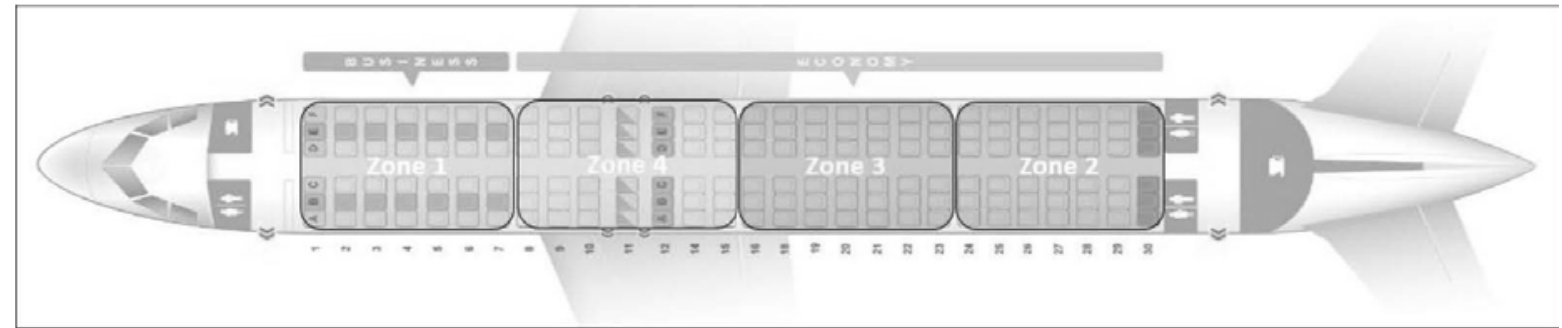

Figure 3: Back-to-front boarding.

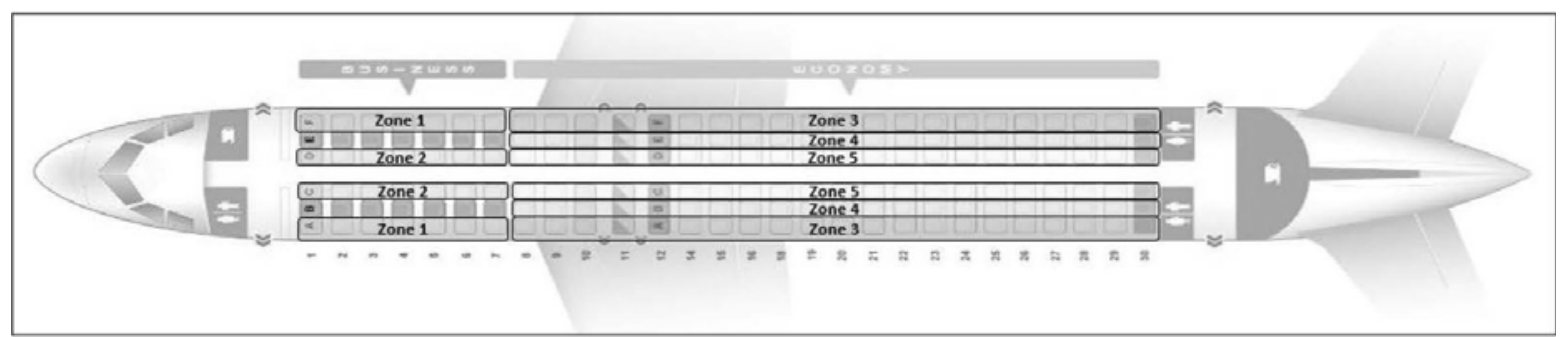

Figure 4: Outside-in boarding.

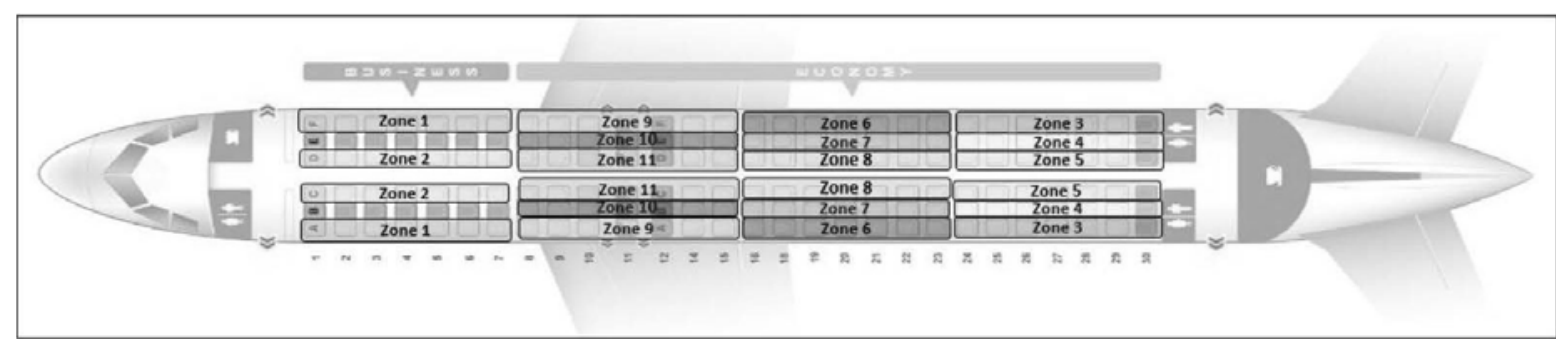

Figure 5: Combination of back-to-front and outside-in boarding. 
This value still means an improvement of one minute and 43 seconds compared to random boarding, but a deterioration of 26 seconds compared to pure outside-in boarding.

Overall, back-to-front boarding is the most inefficient. The main problem is that passengers spend much time waiting in the aisle because a significant number of passengers try to occupy several rows simultaneously. On the one hand, it causes disruption in the row of seats when a passenger who is already seated must get up again because an arriving passenger's seat is at the window or in the middle. On the other hand, only the first passengers arriving at their row of seats can stow away their hand luggage - blocking the way for all following passangers. As a result, the queue is shifted from the gangway into the aircraft. The advantage of this method, like random boarding, seems to be that it is easy to understand, as the aircraft is divided into few areas only where passengers board at the same time.

The advantage of outside-in boarding is that it prevents the aircraft aisle from being congested with passengers blocking the way. In contrast to back-to-front boarding, the distribution regarding the utilization of the aisle is improved (similar to random boarding). In fact, once seated, passengers do not have to stand up again and thus do not block the aisle again. The benefits of boarding methods can be seen in the improved lead time. A decisive disadvantage mentioned in the literature is that the seats in a row are not boarded together. This means that travel groups or families have to separate for a short period of time when boarding the aircraft. Accordingly, acceptance of this boarding method is low, since most passengers assiciate a certain level of comfort with flying. As a result, many airlines discarded this boarding method after a short test phase. A possible solution would be to give families, for example, priority when boarding - similar to business class passengers.

With the combination of the back-to-front and the outside-in boarding method, an attempt is made to combine the advantages of these two methods and to offset their disadvantages. The boarding of the aircraft is done from the back to the front, so that the passengers in the aircraft aisle are as evenly spread as possible. At the same time, however, the aircraft is also boarded from the outside to the inside in order to avoid disturbances within the rows, i.e. row interference. In the present simulation, however, these advantages did not appear at an aircraft utilization of $100 \%$ (but only in further tests with a utilization of $90 \%$ and less).
In addition to this, this method has some practical disadvantages. On the one hand, the correct arrangement of passengers before boarding the aircraft is a challenge, and on the other hand, as already described under the outside-in boarding method, families, or travel groups in general, also have to separate at this point.

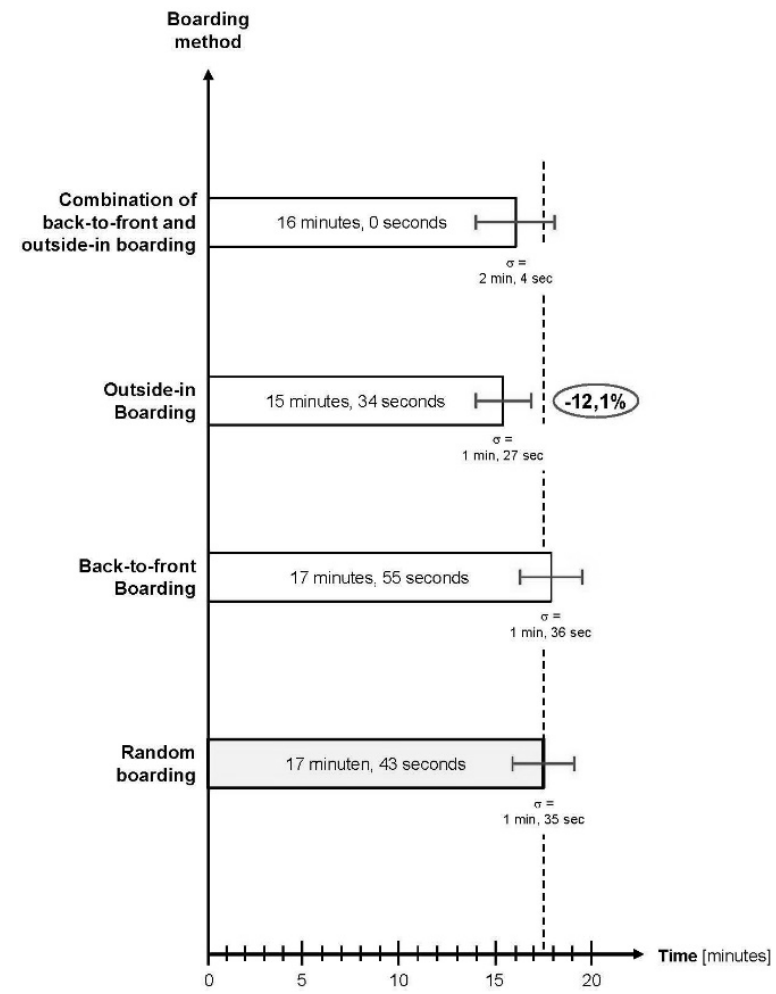

Figure 6: Average boarding duration and standard deviation of the individual boarding methods after 35 simulation runs each.

Should airlines consider changing their current boarding strategy based on the results obtained thus far and with the current situation, the present simulation model offers a good starting point for additional or possible future extensions to approximate the simulated boarding process even closer to reality and hence to determine the optimal boarding strategy for specific environmental or utilization situations. Approaches to possible extensions are, for example, the inclusion of additional boarding methods (e.g. open seating, reverse pyramid, by half block or alternating rows), differentiation according to the number and type of hand luggage in cabin suitcase, hand- or backpack, and jacket including capacity restrictions of the luggage compartments as well as the consideration of different personal walking speeds and an error rate for late passengers. 


\section{Conclusion}

Even with a simple simulation model, it was possible to compare visually the different boarding strategies. What was noticeable was that, at least at a load factor of $100 \%$, the simpler outside-in boarding strategy proved to be more advantageous than the combination of outside-in and back-to-front boarding. The advantage was not only that the average boarding time was 26 seconds shorter, but above all a significantly lower standard deviation of one minute and 27 seconds compared to the standard deviation of two minutes and four seconds for the boarding combination. This increases planning security considerably, which in turn appears to be especially important when the load factor is $100 \%$, which the airlines are aiming for on the few planes currently in use.

In the course of implementation in practice, as a minimum requirement visual aids are needed to increase passengers' understanding of the boarding strategy applied to ensure compliance. Furthermore, it is important to remember that the first prerequisite for a successful realization is that all passengers arrive at the gate at a fixed time at the latest. Since this can almost never be $100 \%$ guaranteed, the implementation of even more sophisticated boarding strategies than those presented in this article or discussed in the academic world will fail.

A representative of Lufthansa even considers boarding to be too complex a process to be able to optimize it with mathematical algorithmic methods alone. Experts who can model and simulate should be brought together with psychologists who can understand and explain group phenomena. In addition to this, cross-cultural differences may be relevant and, for example, cause passengers from group-oriented cultures to be particularly considerate and disciplined during boarding, whereas other cultures impress with punctuality and arrive at the gate on time. In this respect, before refining the simulation model, this begs the question of what effort is involved and what benefits are actually reaped in terms of transferability into practice, unless the prerequisites of the model are already deemed to be too restrictive. Since the crowding in airplanes known from the pre-pandemic era must be avoided during the pandemic, also for reasons of infection control, the new situation now at least offers a chance to experiment with comparatively easy-to-understand out-side-in-boarding.
Note for Publication. This article was originally published in German in ARGESIM Report 59 (ISBN 978-3-901608-93-3). I would like to thank Professor Claudia Wunderlich for editing this English language version.

\section{References}

[1] Airbus. A320 - Aircraft Characteristics Airport and Maintenance Planning; 2018.

[2] Appel H. Analyse der Verzögerungen beim Boarding von Flugzeugen und Untersuchung möglicher Optimierungsansätze [Dissertation]. Rheinisch-Westfälische Technische Hochschule Aachen; 2014

[3] Islam T, Sadeghi Lahijani M, Srinivasan A, Namilae S, Mubayi A, Scotch M. From bad to worse: airline boarding changes in response to COVID-19. Royal Society Open Science 8: 201019; 2021; doi: 10.1098/rsos.201019

[4] Seatguru. https://www.seatguru.com/airlines/Lufthansa/Lufthansa_Airbus_A320-200_NEK.php; (accessed on 10/30/2021)

[5] Slotnick D. The amazing story of how the Airbus A320 became the Boeing 737's greatest rival. Business Insider April 1, 2020; https://www.businessinsider.com/airbusa320-history-boeing-737-rival-2018-9;

(accessed on 10/30/2021) 